\title{
Synchronous primary corpus and ovarian cancer: High incidence of endometriosis and thrombosis
}

\author{
KOJI YAMANOI, MASAKI MANDAI, AYAKO SUZUKI, NORIOMI MATSUMURA, \\ TSUKASA BABA, YUMIKO YOSHIOKA, KENZO KOSAKA and IKUO KONISHI \\ Department of Gynecology and Obstetrics, Graduate School of Medicine, Kyoto University, Kyoto 606-8507, Japan
}

Received March 6, 2012; Accepted June 14, 2012

DOI: 10.3892/ol.2012.770

\begin{abstract}
In an attempt to clarify the clinical characteristics of synchronous primary endometrial and ovarian cancer (SPC), we reviewed the clinicopathological features of 13 cases treated in the Department of Gynecology and Obstetrics at Kyoto University Hospital over the last 6 years and compared them with 186 cases of primary uterine corpus cancer (PCC) and 136 cases of primary ovarian cancer (POC). Comparisons were performed based on clinicopathological factors, including age, BMI, parity, complication of thrombosis and FIGO stage. For SPC patients, the mean age was 51.5 years; $6(46 \%)$ were nulliparous, and 7 (53\%) had complicated thrombosis. All had well-differentiated endometrial cancer and 12 (92\%) had endometrioid cancer in the ovary. The mean age of the SPC patients was significantly lower than that of the PCC patients (51.5 vs. 58.9 years). Thrombosis occurred in the SPC patients at a significantly higher rate than in both the PCC and POC patients. When the incidence of endometriosis and the regularity of menstruation were compared between patients who developed SPC with those who develop PCC at a young age (under 45 years), the SPC patients exhibited a significantly higher rate of endometriosis (100 vs. $35 \%$ ), whereas the PCC patients exhibited a higher rate of irregular menstruation (53 vs. $15 \%, \mathrm{p}=0.05$ ). As for thrombosis, the age and FIGO stage of thrombosis-positive patients were significantly higher than those of thrombosis-negative patients in PCC and POC, while in SPC patients there was no such difference. In conclusion, this study demonstrated the differences in clinical features between SPC and PCC, and also novel features of
\end{abstract}

Correspondence to: Dr Masaki Mandai, Department of Gynecology and Obstetrics, Graduate School of Medicine, Kyoto University, 54 Shogoin Kawahara-cho Sakyo-ku, Kyoto 606-8507, Japan

E-mail: mandai@kuhp.kyoto-u.ac.jp

Key words: synchronous primary endometrial and ovarian cancer, endometriosis, thrombosis, thromboembolism, atypical endometriosis
SPC, namely endometriosis and thrombosis, which are essential in the management of this disease.

\section{Introduction}

Synchronous primary endometrial and ovarian cancer (SPC) is frequently encountered in daily clinical settings. In 1985, Ulbright and Roth first reported the pathological criteria for distinguishing metastatic disease from SPC (1). In 1995, Scully proposed more extensive criteria (2), which are generally used when cancers develop both in the endometrium and in the ovary.

Zaino et al reported that SPC occurs in approximately $10 \%$ of women with ovarian cancer and 5\% of women with endometrial cancer (3). Earlier studies have suggested that there are other unique clinical features in SPC. Soliman et al reported that SPC patients are relatively young and nulliparous. The histology of SPC is mostly well-differentiated endometrioid adenocarcinoma, and its prognosis is better than that of primary uterine corpus cancer (PCC) with ovarian metastasis or primary ovarian cancer (POC) with uterus metastasis (4).

The prevalence of pelvic endometriosis is approximately $6-10 \%$ in women (5). It is estimated that the risk of ovarian cancer is considerably higher in the presence of endometriosis, particularly endometrioid adenocarcinoma and clear cell carcinoma of the ovary $(6,7)$. Furthermore, certain reports have suggested a correlation between endometriosis and SPC (8).

In this study, we reviewed 13 cases of SPC treated at our hospital, and compared their clinicopathological features with those of PCC and POC patients in our department.

\section{Patients and methods}

Patients. This study included 186 patients with PCC, 136 with POC and 13 with SPC. The patients were treated at Kyoto University Hospital from 2005 to 2010. SPC was defined according to the criteria of Scully et al (2). Written informed consent was obtained from each patient.

Demographic data. The following demographic data were obtained from medical records: age at diagnosis, presenting symptoms, body mass index (BMI), parity, past medical history, menopausal status, complication of thrombosis, recur- 


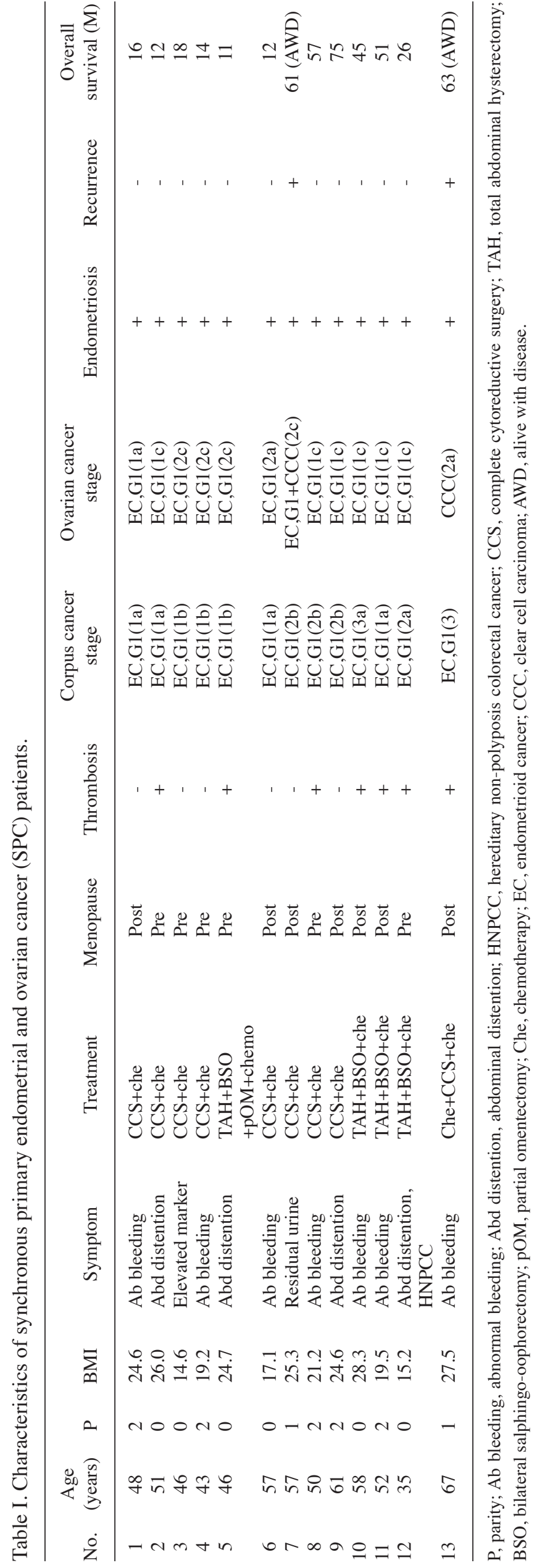

rence of cancer, overall survival rate and menstrual cycle (regular or irregular). Pathological information, including histology, grade and presence of endometriosis, were also obtained. Endometrial and ovarian cancer stages were assigned based on the classification by the International Federation for Gynecology and Obstetrics (FIGO). Histological determination of the endometrial and ovarian cancer was performed based on the World Health Organization (WHO) committee classification of tumors. A comparison of SPC with PCC and POC was performed for several clinical factors including age at diagnosis, BMI, parity and thrombosis complication.

Statistical analysis. The GraphPad prism (MDF Co., Japan) was used for the statistical analyses. Fisher's test was used to assess the significance of differences in the categorical clinical and pathological variables. Continuous variables were analyzed using the Mann-Whitney test. $\mathrm{P}<0.05$ was considered to indicate a statistically significant difference.

\section{Results}

Patient characteristics. The characteristics of the SPC patients are listed in Table I. The mean age at diagnosis was 51.5 years, and the mean BMI was 22. Six of the 13 patients (46\%) were nulliparous. Histologically, the lesion in the endometrium was endometrioid adenocarcinoma (G1/G2) in all cases, whereas the lesion in the ovary was well-differentiated endometrioid adenocarcinoma in 12 of 13 cases, and clear cell carcinoma in one case. The age at SPC diagnosis was significantly lower than the age at PCC diagnosis (51.5 vs. 58.9 years; Table II). A greater number of SPC patients were nulliparous (46.2\%) compared with the PCC (28.5\%) and POC (33.8\%) patients, although there was no significant difference. In our department, complete cytoreductive surgery with adjuvant chemotherapy is the standard treatment course for SPC. Recurrent disease was observed in two SPC patients. All patients, including those with disease recurrence, were alive as of January 2012. Endometriosis was observed in all of the SPC cases.

Comparison of young PCC and SPC patients. As the age of the SPC patients was significantly lower than that of PCC patients, we selected the young PCC patients (those under 45 years old) with G1 and G2 endometrioid adenocarcinoma, and compared them with the SPC patients in terms of the clinicopathological factors. The POC patients whose histological type was endometrioid adenocarcinoma or clear cell carcinoma were also used for comparison (Table III). There was no significant difference between each group in terms of age, BMI and nulliparity. However, an irregular menstrual cycle was more frequently observed among the PCC patients under 45 years old than among the SPC patients (9 of 17, $52.9 \%$ vs. 2 of 13, $15.4 \%)$, although the difference was not significant $(\mathrm{p}=0.05)$. In addition, endometriosis complications were more frequent among the SPC patients than the PCC patients under 45 years (17 of 17, 100\% vs. 6 of 17,35.3\%). Moreover, the incidence of thrombosis was significantly higher in the SPC patients (7 of $13,53.8 \%$ vs. none, $0 \%)$.

Incidence of thrombosis. Thrombosis complications were observed in SPC patients at a significantly higher frequency 
Table II. Comparison of clinicopathological features among SPC, POC and PCC patients.

\begin{tabular}{|c|c|c|c|}
\hline & $\mathrm{SPC}(\mathrm{n}=13)$ & POC $(n=136)$ & $\operatorname{PCC}(n=186)$ \\
\hline Age (years) & 51.5 & 54.5 & $58.9^{\mathrm{a}}$ \\
\hline \multicolumn{4}{|l|}{ Type of cancer } \\
\hline Endometrioid adenocarcinoma G1/G2 (\%) & $13(100)$ & $11(8.1)$ & $101(54.5)$ \\
\hline Endometrioid adenocarcinoma G3 (\%) & & $6(4.4)$ & $34(18.2)$ \\
\hline Serous adenocarcinoma $(\%)$ & & $53(39.0)$ & $31(16.6)$ \\
\hline Clear cell carcinoma $(\%)$ & $1(7.8)$ & $37(27.2)$ & $8(4.3)$ \\
\hline Mucinous adenocarcinoma (\%) & & $15(11.0)$ & $1(0.5)$ \\
\hline Others $(\%)$ & & $24(17.6)$ & $10(5.3)$ \\
\hline \multicolumn{4}{|l|}{ Stage } \\
\hline 1 & $1+1: 3$ cases & $64(47.1)$ & $111(59.4)$ \\
\hline 2 & $\begin{array}{c}2+1: 3 \text { cases, } 1+2: 4 \text { cases, } \\
2+2: 1 \text { cases }\end{array}$ & $5(3.7)$ & $14(7.5)$ \\
\hline 3 & $3+1: 1$ case $, 3+2: 1$ case & $47(34.6)$ & $46(24.6)$ \\
\hline 4 & & $18(13.2)$ & $16(8.6)$ \\
\hline $\mathrm{BMI}\left(\mathrm{kg} / \mathrm{m}^{2}\right)$ & 22.1 & 21.9 & 23.5 \\
\hline Nulliparity (\%) & $6(46.2)$ & $46(33.8)$ & $54(28.9)$ \\
\hline Thromboembolism (\%) & $7(53.8)^{*}$ & $20(14.7)$ & $10(5.3)$ \\
\hline
\end{tabular}

${ }^{\mathrm{a}} \mathrm{p}<0.05$. For SPC patients, the stage is given as corpus cancer stage first and ovary cancer stage second. Therefore, $1+1$ means corpus cancer is stage 1 (within corpus body), and ovarian cancer is stage 1 (within unilateral side). SPC, synchronous primary endometrial and ovarian cancer; POC, primary ovarian cancer; PCC, primary uterine corpus cancer.

Table III. Comparison among patients with SPC, PCC with endometrial cancer (G1/G2 under 45 years old), POC with ovarian endometrioid adenocarcinoma and POC with clear cell carcinoma, which are recognized as endometriosis-related cancer.

\begin{tabular}{|c|c|c|c|c|}
\hline & $\begin{array}{c}\text { SPC } \\
(n=13)\end{array}$ & $\begin{array}{c}\text { PCC } \\
\text { (em cancer } \mathrm{G} 1 / \mathrm{G} 2, \\
\text { under } 45 \text { years; } \mathrm{n}=17)\end{array}$ & $\begin{array}{c}\text { POC } \\
\text { (endometrioid cancer; } \\
n=17 \text { ) }\end{array}$ & $\begin{array}{c}\text { POC } \\
\text { (clear cell carcinoma; } \\
n=37)\end{array}$ \\
\hline Age (years) & 51.5 & & 55.1 & 53.1 \\
\hline \multicolumn{5}{|l|}{ Stage $(\%)$} \\
\hline 1 & $1+1: 3$ cases & $15(88.2)$ & $10(58.8)$ & $28(75.7)$ \\
\hline \multirow[t]{2}{*}{2} & $2+1: 3$ cases, $1+2: 4$ cases & & & \\
\hline & $2+1: 1$ case & $1(5.9)$ & $1(5.9)$ & $1(2.7)$ \\
\hline 3 & $3+1: 1$ cases, $3+2: 1$ case & $1(5.9)$ & $5(29.4)$ & $4(10.8)$ \\
\hline 4 & & $0(0)$ & $1(5.9)$ & $4(10.8)$ \\
\hline BMI $\left(\mathrm{kg} / \mathrm{m}^{2}\right)$ & 22.1 & 21.9 & 24.3 & 21.7 \\
\hline Nulliparity (\%) & $6(46.2)$ & $13(76.5)$ & $6(35.3)$ & $20(54.1)$ \\
\hline Thromboembolism (\%) & $7(53.8)^{\mathrm{a}}$ & $0(0)$ & $1(5.9)$ & $6(16.2)$ \\
\hline Irregular mense $(\%)$ & $2(15.4)^{\mathrm{b}}$ & $9(52.9)$ & & \\
\hline Endometriosis (\%) & $13(100)^{\mathrm{a}}$ & $6(35.3)$ & & \\
\hline
\end{tabular}

${ }^{\mathrm{a}} \mathrm{p}<0.05 ;{ }^{\mathrm{b}} \mathrm{p}=0.05$. For SPC patients, the stage is given as corpus cancer stage first and ovary cancer stage second. Therefore, $1+1$ means corpus cancer is stage 1 (within corpus body), and ovarian cancer is stage 1 (within unilateral side). SPC, synchronous primary endometrial and ovarian cancer; POC, primary ovarian cancer; PCC, primary uterine corpus cancer; em cancer, endometrial cancer.

(7 of $13,53.8 \%$ ) than in PCC patients (10 of 186, 5.3\%) and POC patients (20 of 146, 14.7\%). We further investigated the clinical features of the thrombosis cases (Tables IV and V). Each group of cancer patients was divided into a thrombosis
(+) group and a thrombosis (-) group. Among the PCC patients, patients with thrombosis were significantly older and had a more advanced stage of disease (FIGO stage III+IV) compared with those without thrombosis. POC patients with thrombosis 
Table IV. Comparison among thrombosis(+) patients in each group.

\begin{tabular}{lccc}
\hline & SPC $(\mathrm{n}=7)$ & POC $(\mathrm{n}=20)$ & PCC $(\mathrm{n}=10)$ \\
\hline $\begin{array}{l}\text { Age (years) } \\
\text { Type of cancer }\end{array}$ & $51.1^{\mathrm{a}}$ & 62.8 & 66.1 \\
Endometrioid adenocarcinoma G1/G2 (\%) & & & $3(30)$ \\
Endometrioid adenocarcinoma G3 (\%) & $7(100)$ & $1(0.5)$ & $1(10)$ \\
Serous adenocarcinoma (\%) & & $10(50.0)$ & $4(40)$ \\
Clear cell carcinoma (\%) & $1(28.6)$ & $6(30.0)$ & $1(10)$ \\
Mucinous adenocarcinoma (\%) & & $2(10.0)$ & $1(10)$ \\
Others $(\%)$ & & $3(15.0)$ & $2(20)$ \\
Stage & $1+1: 2$ cases & & $1(10)$ \\
1 & $2+1: 2$ cases, $1+2: 1$ case & $1(0.5)$ & $3(30)$ \\
2 & $3+1: 1$ cases, $3+21$ case & $6(30.0)$ & $4(40)$ \\
3 & & $7(35.0)$ & 22.8 \\
4 & 23.2 & 22.5 & $2(20)$ \\
BMI $\left(\mathrm{kg} / \mathrm{m}^{2}\right)$ & $4(66.2)$ & $3(15.0)$ & \\
Nulliparity $(\%)$ & & & \\
\hline
\end{tabular}

${ }^{\mathrm{a}} \mathrm{p}<0.05$. For SPC patients, the stage is given as corpus cancer stage first and ovary cancer stage second. Therefore, $1+1$ means corpus cancer is stage 1 (within corpus body), and ovarian cancer is stage 1 (within unilateral side). SPC, synchronous primary endometrial and ovarian cancer; POC, primary ovarian cancer; PCC, primary uterine corpus cancer.

were also significantly older than those without thrombosis. In contrast, there was no significant difference in age and FIGO stage between the SPC patients with and without thrombosis. In addition, when the incidence of thrombosis was compared in advanced stage patients (FIGO stage II+III ovarian cancer or FIGO stage III endometrial cancer), the SPC patients had a significantly higher rate of thrombosis than the PCC $(\mathrm{p}<0.001)$ or POC $(\mathrm{p}=0.004)$ patients.

\section{Discussion}

In this study, we compared several clinicopathological features of SPC with those of PCC or POC. First, we found a significant difference in the age at diagnosis between patients with SPC and those with PCC. Patients with SPC were significantly younger than those with PCC (51.5 vs. 58.9 years). Additionally, the mean age of patients with SPC was relatively lower compared to POC, although the difference was not statistically significant. In addition, patients with SPC exhibited a relatively high rate of nulliparity compared to those with PCC. There are several earlier reports that describe the clinicopathological features of SPC. In their review of 84 cases of SPC, Soliman et al reported that women with SPC were young, obese and nulliparous (4). Sultan et al also indicated that women with SPC were significantly younger than women with PCC and POC (9). Herrinton et al reported that high parity and long-term use of oral contraceptives reduced the risk of SPC (10). Taken together, younger age and nulliparity appear to be unique features of SPC. Our next question was whether the difference between SPC and PCC is due to the inclusion of older PCC patients, who might be significantly different from younger patients in terms of tumor biology as well as host condition. To address this issue, we selected only the young PCC patients under the age of 45 .
When the SPC patients were compared with this group of PCC patients, there were still significant differences: the incidence of endometriosis (100 vs. 35\%) and thrombosis (54 vs. 0\%) was significantly higher in SPC patients. These data suggest that SPC has several unique clinical features that are distinct from PCC in patients of a similar age.

In all 13 SPC cases, the lesion in the endometrium was well-differentiated endometrioid carcinoma, and in 11 of the 13 cases, the lesion in the ovary was also well-differentiated endometrioid carcinoma. Of the other two SPC cases, one was clear cell carcinoma, and the other involved both well-differentiated endometrioid carcinoma and clear cell carcinoma in the ovary. According to previous reports, the majority of SPC cases consist of well-differentiated endometrioid carcinoma in both the endometrium and the ovary $(4,9,11)$, which is consistent with the results of the present study. In a relatively short follow-up period, only $2(15.4 \%)$ cases of SPC recurred, and both patients are currently alive, suggesting a favorable outcome of SPC. Most of the previous reports refer to an excellent overall survival rate in women with SPC, which is reportedly due to the earlier stage at diagnosis of SPC compared with POC. The first symptom in the majority of SPC cases is abnormal bleeding (4). Accordingly, in our study, 8 of the 13 SPC patients visited our hospital due to abnormal bleeding. However, Williams et al revealed that SPC still had a better prognosis that was independent of stage, even after adjusting for other prognosis factors. In other words, SPC itself might be directly associated with good prognosis (12).

Notably, in our study, the presence of endometriosis was a factor in all the SPC patients. Endometriosis represents a significant site of origin of ovarian cancer, particularly in the case of endometrioid adenocarcinoma and clear cell carcinoma. Several previous reports have suggested a correlation between 
Table V. Comparison of clinical features of thrombosis.

A, Comparison of age between patients with positive and negative thrombosis status in each group

\begin{tabular}{lccc}
\hline & Thrombosis $(+)$ & Thrombosis(-) & p-value \\
\hline SPC & 51.1 & 52.0 & 1.0 \\
POC & 62.8 & 53.0 & 0.002 \\
PCC & 66.1 & 58.0 & 0.03 \\
\hline
\end{tabular}

$\mathrm{B}$, Comparison of ratio of thrombosis(+) between patients with low-stage and high-stage disease in each group

\begin{tabular}{lccc}
\hline & Thrombosis $(+) /$ stage $1+2$ & Thrombosis $(+) /$ stage $3+4$ & p-value \\
\hline SPC $^{\text {a }}$ & $5 / 11$ & $2 / 2$ & 0.46 \\
POC & $7 / 69$ & $13 / 65$ & 0.15 \\
PCC & $3 / 125$ & $7 / 60$ & 0.015 \\
\hline
\end{tabular}

C, Comparison of positive vs. negative thrombosis ratio among patients with SPC, POC stage $2+3$ and PCC stage 3

\begin{tabular}{lcc}
\hline & Thrombosis $(+)$ & Thrombosis(-) \\
\hline SPC & $7 / 13$ & $6 / 13^{\text {b,c }}$ \\
POC stage 2+3 & $7 / 52$ & $45 / 52$ \\
PCC stage 3 & $3 / 46$ & $43 / 46$ \\
\hline
\end{tabular}

${ }^{\mathrm{a}}$ For SPC patients, corpus cancer stage is used to divide the two groups. ${ }^{b} \mathrm{p}=0.0042$ compared with POC stage $2+3$. ${ }^{\mathrm{c}} \mathrm{p}=0.0004$ compared with PCC stage 3. SPC, synchronous primary endometrial and ovarian cancer; POC, primary ovarian cancer; PCC, primary uterine corpus cancer.

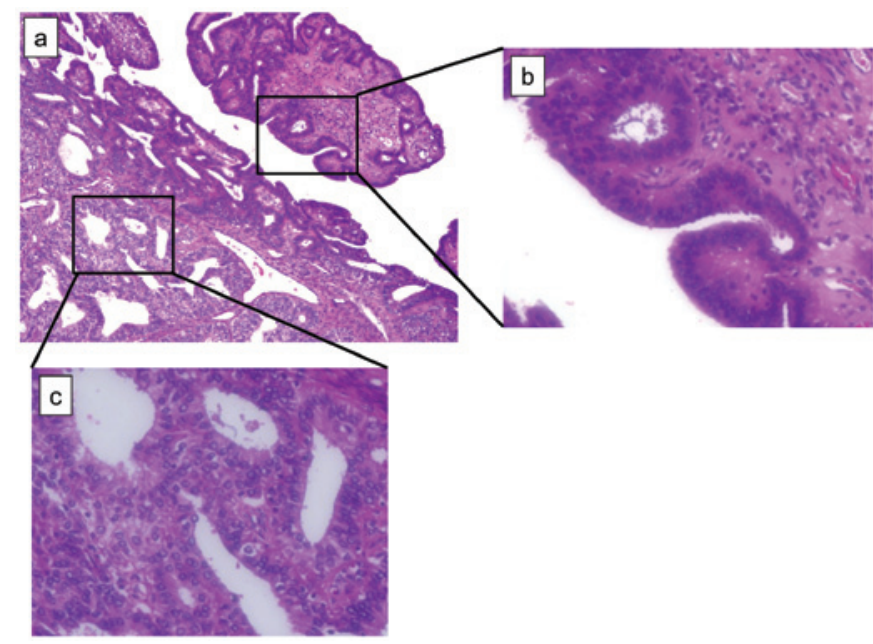

Figure 1. Microscopic view of ovary lesion of patient no. 7. (a) Both atypical endometriosis (right side) and endometrioid adenocarcinoma (left side) co-exist (10x10). (b) Magnification of right black square in (a); atypical endometriosis (10x40). (c) Magnification of left black square in (a); welldifferentiated endometrioid adenocarcinoma (10x40).

endometriosis and SPC. Kondi-Pafiti et al pathologically confirmed endometriosis in all patients with SPC (13). Zaino et al reported the presence of endometriosis in $31 \%$ of SPC patients (3). Two possibilities have been suggested regarding the association between endometriosis and SPC. First, endometriotic implants may undergo direct malignant transformation, often through atypical endometriosis. Second, cancer and endometriosis share various environmental, immunological, hormonal and genetic predisposing factors (14). In our study, the clinicopathological features (irregular menstruation cycle and presence of endometriosis) of SPC resemble those of endometriosis-associated ovarian cancer rather than young-age PCC. In some of our SPC patients, atypical endometriosis was observed (Fig. 1), which is reportedly found in approximately $60-80 \%$ of endometriosis-associated ovarian cancer $(8,14)$.

Cancer-related thrombosis is also known as Trousseau's syndrome, which was first reported in 1865 in association with gastric cancer (15). Factors secreted by cancer cells impair the coagulation and fibrinolytic system. One of these factors is tissue factor (TF) (16). Conversion of factor VII to its active form (factor VIIa) in complex with TF triggers the production of other coagulation-related proteases, particularly factor $\mathrm{X}$ and factor IXa. Elevated TF expression is observed in carcinoma cells in Trousseau's syndrome as well as in associated angiogenic endothelium. Activated oncogenes (K-ras, EGFR, PML-RARA and MET) or inactivated tumor suppressors (p53 and PTEN) are known to lead to an induction of TF and its activity (17). It is also suggested that tumor hypoxia increases the expression of genes that facilitate coagulation, including TF and plasminogen activator inhibitor type 1 (PAI-1) (18). In gynecological malignancies, ovarian cancer, particularly clear cell carcinoma, is known to increase the development of complicated thrombosis $(19,20)$. Uno et al studied TF activity in ovarian cancer, and reported that TF expression 
in clear cell carcinoma is significantly increased compared with that in non-clear cell carcinoma (21). We demonstrated that clear cell carcinoma of the ovary has aberrant expression of coagulation-related genes, which is caused by the microenvironment within the endometriotic cyst (22). Thus far, no study has addressed the association between SPC and thrombosis. In the current study, patients with SPC developed thromboses at a significantly higher rate compared to PCC and POC patients; as many as 53.8\% (7 of 13) of the SPC patients developed complicated thrombosis prior to starting therapy. In the PCC and POC patients, those who developed thrombosis were significantly older than those who did not. In patients with PCC, those who developed thrombosis had a significantly higher FIGO stage than those who did not. However, in SPC cases, there was no such tendency. Although there was only a relatively small number of cases included in this study, SPC itself may be an independent risk factor for thrombosis, similar to clear cell carcinoma.

In conclusion, this study indicates that SPC has several unique features, including young age, nulliparity and a better prognosis. In addition, endometriosis was detected in all of the SPC patients, which is consistent with several previous studies. Clinically, SPC may be more similar to endometriosisrelated ovarian cancer than young-age corpus cancer. Notably, thrombosis occurred in patients with SPC at a significantly higher rate than in PCC and POC. There may be specific carcinogenetic mechanisms in SPC that are related to its own unique features, including the co-existence of endometriosis and thrombogenic tendency. Further research is required to explore these mechanisms.

\section{References}

1. Ulbright T and Roth L: Metastatic and independent cancers of the endometrium and ovary: a clinicopathologic study of 34 cases. Hum Pathol 16: 28-34, 1985.

2. Scully RE, Young RH and Clements PB: Tumors of the ovary, maldeveloped gonads, fallopian tube, and broad ligament. In: Atlas of Tumor Pathology. Series 3, Fascicle 23. Rosai J and Sobin LH (eds). Armed Forces Institute of Pathology, Washington DC, pp125-126, 1998 .

3. Zaino R, Whitney C, Brady MF, et al: Simultaneously detected endometrial and ovarian carcinomas- a prospective clinicopathologic study of 74 cases: a Gynecologic Oncology Group study. Gynecol Oncol 83: 355-362, 2001.
4. Soliman PT, Slomovitz BM, Broaddus RR, Sun CC, Oh JC, Eifel PJ, Gershenson DM and Lu KH: Synchronous primary cancers of the endometrium and ovary: a single institution review of 84 cases. Gynecol Oncol 94: 456-462, 2004.

5. Giudice LC and Kao LC: Endometriosis. Lancet 364: 1789-1799, 2004.

6. Komiyama S, Aoki D, Tominaga E, et al: Prognosis of Japanese patients with ovarian clear cell carcinoma associated with pelvic endometriosis: Clinicopathologic evalution. Gynecol Oncol 72: 342-346, 1999.

7. McMeekin DS, Burger RA, Manetta A, et al: Endometrioid adenocarcinoma of the ovary and its relationship to endometriosis. Gynecol Oncol 59: 81-86, 1995.

8. Stern RC, Dash R, Bentley RC, et al: Malignancy in endometriosis: frequency and comparison of ovarian and extraovarian types. Int J Gynecol Pathol 20: 133-139, 2001.

9. Sultan E, Ibrahim G, Raziye O, et al: Synchronous primary cancers of the female reproductive tract in Turkish women. Asian Pac J Cancer Prev 12: 857-859, 2011.

10. Herrinton LJ, Voigt LF and Weiss NS: Risk factors for synchronous primary endometrial and ovarian cancers. Annals of Epidemiology 11: 529-533, 2001.

11. Lim YK, Padma R, Foo L, et al: Survival outcome of women with synchronous cancers of endometrium and ovary: a 10 year retrospective cohort study. J Gynecol Oncol 22: 239-243, 2011.

12. Williams MG, Bandera EV, Demissie K, et al: Synchronous primary ovarian and endometrial cancers. A population-based assessment of survival. Obstet Gynecol 113: 783-789, 2009.

13. Kondi-Pafiti A, Grapsa D, Liapsi K, et al: Synchronous ovarian and endometrial carcinoma: a strong link to endometriosis? Eur J Gynaecol Oncol 29: 256-259, 2008.

14. Varma R, Rollason T, Gupta JK, et al: Endometriosis and the neoplastic process. Reproduction 127: 293-304, 2004.

15. Donati MB. Thrombosis and cancer: Trousseau syndrome revisited. Best Pract Res Clin Haematol 22: 3-8, 2009.

16. Varki A: Trousseau's syndrome: multiple definitions and multiple mechanisms. Blood 110: 172-1729, 2007.

17. Rak J, Yu JL, Luyendyk J, et al: Oncogenes, Trousseau syndrome, and cancer-related changes in the coagulome of mice and humans. Cancer Res 66: 10643-10646, 2006.

18. Denko NC and Giaccia AJ: Tumor hypoxia, the physiological link between Trousseau's syndrome (carcinoma-induced coagulopathy) and metastasis. Cancer Res 61: 795-798, 2004.

19. Heit JA, Silverstein MD, Mohr DN, et al: Risk factors for deep vein thrombosis and pulmonary embolism. Arch Intern Med 160: 809-815, 2000.

20. Matsuura Y, Robertson G, Marsden DE, et al: Thromboembolic complications in patients with clear cell carcinoma of the ovary. Gynecol Oncol 104: 406-410, 2007.

21. Uno K, Homma S, Satoh T, et al: Tissue factor expression as a possible determinant of thromboembolism in ovarian cancer. $\mathrm{Br}$ J Cancer 96: 290-295, 2007.

22. Yamaguchi K, Mandai M, Oura T, et al: Identification of ovarian clear cell carcinoma gene signature that reflects inherent disease biology and carcinogenic processes. Oncogene 29: 1741-1752, 2010. 